\title{
Atmospheric Refractivity Estimation from Radar Sea Clutter Using Novel Hybrid Model of Genetic Algorithm and Artificial Neural Networks
}

\author{
Cemil TEPECIK, Isa NAVRUZ, O. Tolga ALTINOZ \\ Department of Electrical and Electronics Engineering, Ankara University, 06100, Ankara, Turkey \\ \{ctepecik, Isa.Navruz, Tolga.Altinoz\}@ankara.edu.tr \\ Submitted February 5, 2020 / Accepted June 13, 2020
}

\begin{abstract}
This paper is focused on solving the inversion problem of refractivity from clutter (RFC) technique. A novel hybrid model is developed that can estimate the atmospheric refractivity ( $M$ profile) with a high accuracy, for surface based duct case, which is most effective nonstandard propagation condition on radar observation. The model uses propagation factor curve in horizontal axis, whose characteristics is determined by $M$ profile for estimation. The model is based on artificial neural network, which includes a dynamic training data approach, and a problem adapted genetic algorithm. Dynamic training data set application is a nonstandard approach in neural network applications, in which every obtained result are dynamically added to data set during the estimation process, for a better estimation. Firstly, neural network and genetic algorithm have been adapted to the characteristics of inversion problem separately. Then, the mentioned two methods have been harmonized and run together. Ultimately, the final algorithm has evolved into a complex adapted hybrid model, which is easily applicable to clutter data obtained by any real radar from the real environment. The results show that the proposed model presents considerably effective solution to refractivity estimation problem.
\end{abstract}

\section{Keywords}

Hybrid intelligent systems, radio wave propagation, surface based duct, parameter estimation

\section{Introduction}

Prediction of radar coverage is a critical issue for military maritime and air surveillance. Radar coverage programs need atmospheric refractive index structure within radar range to produce a precise coverage. There are many alternative methods to determine refractive indexes such as radiosonde balloon, rocketsonde, lidar and refractometer. However, these methods have some advantages and disadvantages concerning the accuracy, time, difficulty and cost [1]. Refractivity from clutter (RFC) is another prediction method where radar itself is used as a transducer and does not require any additional measurement equipment. Clutter is normally an unwanted portion of the received signal, but it is useful to infer the atmospheric environment [2]. RFC has two main branches: An electromagnetic (EM) wave propagation model and a refractivity estimation model [3]. When the atmospheric conditions allow for surface ducting, the distinct clutter rings appear on the radar screen and the radar range of detection surprisingly extends beyond the line of sight [4]. This clutter rings or clutter return locations give a clue about the atmospheric refractivity profile, which determines characteristic of duct, especially the surface based duct (SBD) which is the most effective duct type over radars.

Therefore, with the aid of previous discussion and experimental results, this study focuses on the prediction of refractivity profile ( $\mathrm{M}$ profile). In addition as a novel contribution; a hybrid model for solving complex inversion problem coming from RFC method is proposed and discussed in detail. In the context of this paper, an Artificial Neural Network (ANN), two different Genetic Algorithms (GA); one is standard (sGA), and the other is problem adapted (aGA), and two different Hybrid Models (HM) consisting of a combination of GAs and ANN are introduced. One of the hybrid models is standard (sHM), and the other is problem adapted (aHM). GA is one of the popular optimization algorithms for the solution of the refractivity estimation problem, and it was firstly used by Gerstoft et al. [4]. Gerstoft prepared a software package based on Simulated Annealing/Genetic Algorithm for inversion problems previously [5]. The basic concepts of electromagnetic matched-field processing and the related GAs based on global optimization procedures are theoretically discussed by Gerstoft et al. [6]. In another study, a hybrid model of genetic algorithm and Markov Chain Monte Carlo method were used for statistical maritime radar duct estimation problem [7]. Zhao organized the RFC problem as a multi-parameter optimization issue and used the genetic algorithm for height estimation of evaporation duct and source localization [8]. In 2014, Grimes and Hackett used the GA as an inversion method examining constants of an evaporation duct model [9]. ANN is a computational model based on the structure and functions of biological neural 
networks, and it was applied to this kind of problems recently. Hosseinzadeh et al. proposed a network that consists of two cascade neural networks to determine the tropospheric refractivity slop [10]. Mudroch used the ANN for remote sensing of the atmosphere and classification and prediction of the lower troposphere layers influence on RF propagation [11]. Da Silveira and Holt used the neural networks for automatic identification of clutter and anomalous propagation in polarization-diversity weather radar data [12]. Also, Grecu and Krajewski applied ANN for detection of anomalous propagation echoes [13]. Pelliccia et al. developed an ANN algorithm as a useful tool for determination of Arctic atmosphere sounding [14]. Tepecik and Navruz introduced a model based on ANN and GA for solving the inversion problem of refractivity estimation [15].

In our previous study [15], in order to estimate the $\mathrm{M}$ profile of the atmosphere from propagation factor curve, obtained from sea surface level radar clutter data, a cascade model of ANN and GA was presented where GA used the results obtained by the ANN only once. In this study, we propose a couple of novel hybrid models in which GA and ANN are designed to collaborate dynamically and have a potential of providing high performance. Here, ANN and GA receive the previously obtained estimations dynamically in each iteration and use them for the next iteration. GA side of hybrid model executes the first estimations, and then these estimations are added to the ANN training data set. The estimations produced by ANN side are transferred to GA and GA produces next iterations using the qualified ANN results. These processes are repeated for both sides of hybrid structure until the satisfied estimation is obtained. The ANN structure used in this new hybrid model is improved in several ways compared to that used in [16]. First of all, a bigger data set is used, which is elevated from 707 to 5488 in number. Secondly, in the process of atmospheric refraction estimation, new estimates of the hybrid model are continuously added to the ANN dynamic training data set as new data. It was a static data used in previous works for ANN training. Finally, a more complex ANN structure has been designed to achieve faster predictive results with targeted accuracy. Another main difference between the new study and the previous one comes from the increment of sample data representing the EM propagation factor curve. Number of sample is 144 in this study, while it was 25 in [16]. The new model is explained in the following sections in detail and benefits of the novel approach are also proved with obtained results.

The paper is organized as follows: Section 2 gives the theory of surface-based ducts and tri-linear refractivity profile. Section 3 presents the EM propagation model, analysis for the inversion problem, simulation data and success criteria of the algorithms. Section 4 is allocated for inversion problem by using ANN and GA techniques and evaluation of their refractivity profile estimation performances. In the last section, the proposed hybrid models with two different approaches (standard and adapted) are demonstrated, and all results are compared and discussed.

\section{Theory of Surface-Based Ducts}

Two atmospheric factors can lead to electromagnetic ducts; one is a humidity inversion, where the water-vapor content decreases with height, and the other is a temperature inversion, where the temperature increases with height [1]. Ducts can trap electromagnetic signals, so they cause them propagate longer distances than usual. SBD is one of the three types of ducts. Other duct types are evaporation duct and elevated duct. Atmospheric index of refraction $n$ determines duct characteristics. As a variable of time and height, the value of $n$ is slightly different from one (1.00025-1.00040) around Earth's surface [17]. Atmospheric refractivity $N$ is computed as

$$
N=\frac{77.6 p}{T}-\frac{5.6 e}{T}+\frac{3.75 \times 10^{5} e}{T^{2}}
$$

where $p$ is the air pressure $(\mathrm{hPa}), T$ is the absolute temperature $(\mathrm{K})$, and $e$ is the water vapor pressure $(\mathrm{hPa})$, which is obtained from the relative humidity. To identify ducting conditions, it is convenient to use a modified index of refraction defined as $M=N+0.157 h$, where $h$ is the height above the surface. The EM duct occurs when [18]

$$
\frac{\mathrm{d} M}{\mathrm{~d} h}<0 \quad \text { or } \quad \frac{d N}{\mathrm{~d} h}<-157
$$

The relationship between duct thickness and radar frequency is formulated by Cairns-McFeeters [19] as follows

$$
f_{\min }=3.6 \times 10^{11} d^{-3 / 2}
$$

where $f_{\min }$ is the minimum frequency and $d$ is the duct thickness. RFC technique is not useful for prediction of elevated ducts since this type of duct does not create enough clutter on radar screen. In addition, not all SBDs can be estimated by using RFC technique [20]. Recently, a study about sea surface-EM wave propagation interaction and effects on the usage of RFC technique is carried out [21]. Interaction of the EM wave and sea is a multidimensional problem. It depends on meteorology, the altitude of the signal source, frequency and polarization. In this work, we focused on horizontally polarized L band radar to examine SBD estimation.

In many cases, a simple tri-linear M-profile is used to describe a SBD. There are 5 main parameters to characterize

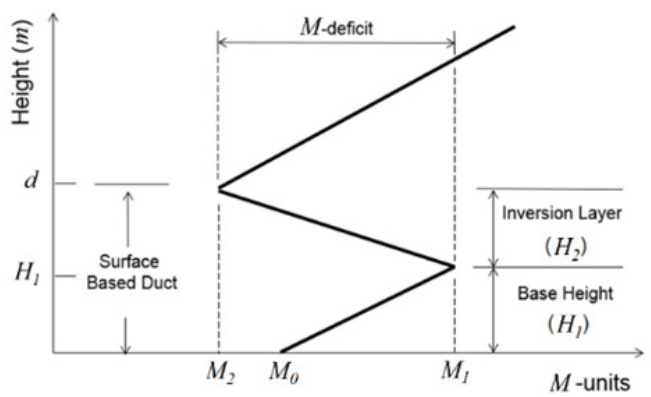

Fig. 1. Tri-linear profile for modified refractivity. 
tri-linear M-profile SBD as shown in Fig. 1. Three of them are modified refractivity parameters in M-unit $\left(M_{0}, M_{1}\right.$ and $M_{2}$ ). M-deficit is defined as the difference of $M_{1}$ and $M_{2}$. $M_{0}$ is the surface level modified refractivity. $H_{1}$ is the base height, and $\mathrm{H}_{2}$ is the thickness of trapping layer. The sum of $H_{1}$ and $H_{2}$, represented by $d$ in (3), is the total thickness of the SBD channel. The M-profile is assumed rangeindependent, and the tri-linear profile is used to model the vertical M-profile or SBD in the study.

\section{Method}

Radars, operating in maritime environments, receive a back-scattered signal from the sea surface. The received clutter signal depends on the refractivity profile of the atmospheric environment known as M-profile [22]. Received signal power $P_{\mathrm{c}}$ from the clutter at the range $r$ is computed from the radar equation:

$$
P_{\mathrm{c}}(r)=\frac{P_{\mathrm{t}} G^{2} \lambda^{2} \sigma_{\mathrm{c}} F^{4}(r, m)}{(4 \pi)^{3} r^{4} L}
$$

where $P_{\mathrm{t}}$ is the transmitter power, $G$ is the antenna gain, $\lambda$ is the wavelength, $\sigma_{\mathrm{c}}$ is the clutter cross-section, $L$ is the total assumed system loss, $F(r, m)$ is the propagation factor (PF) and $m$ is the refractivity profile in a maritime environment. The PF is defined as the ratio of the magnitude of the electric field at a given point under specified conditions to the electric field under free-space conditions [17]. The coverage of the radar system is simulated and analyzed with the aid of PETOOL software, which is a problemoriented program. This software uses a split step parabolic equation (SSPE) approach with wide-angle propagator method and presents radar coverage regarding PF [23]. SSPE is an initial value problem starting from a reference point and marching out in range by obtaining the field along the vertical direction at each range step through the use of step-by-step Fourier transformations.

Inversion Problem of RFC Method: The estimation problem of the RFC method is the process of obtaining the vertical atmospheric refractivity parameters from the measured radar clutter data. Models, such as SSPE, form propagation patterns using atmospheric refractivity parameters. On the other hand, obtaining the vertical refractivity profile from the propagation pattern is a non-linear inverse process. Many methods have been used for the solution of inversion problem in atmospheric refractivity estimation. Some of the methods used are: matched-field processing approach toward inversion [6], genetic algorithm [4], Markov-chain Monte Carlo sampling approach [7], Kalman and particle filters [2], support vector machines [24], particle swarm optimization [25], improved best fit approach [26], adaptive objective function [27] and neural network [16], [28]. The main utility of RFC is that the strongest clutter returns come from regions where radar illumination is most powerful. The purpose of inversion is to determine the best SBD parameters that match with observed clutter map. If the radar parameters are known in (4), sea surface reflec- tivity $\sigma^{0}$ can be modeled correctly. In this case, real PF values can be computed easily from the radar clutter equation, which can be written in $\mathrm{dB}$ as follows:

$$
\begin{aligned}
P_{\mathrm{c}}(r)_{\mathrm{dB}}= & 10 \log \left(\frac{P_{\mathrm{t}} G^{2} \lambda^{2}}{(4 \pi)^{3} L}\right)-40 \log (r)+10 \log \left(\sigma_{\mathrm{c}}\right) \\
& +40 \log (F(r, m)) .
\end{aligned}
$$

Range value is known since it can be measured by radar. Therefore, the signal power detected by the radar at any specific $r$ distance is $P_{\mathrm{c}}(r)$. In this case, the first and second terms, which are radar parameters and range value, are known in (5). If $C_{0}$ denotes all known values, $\mathrm{PF}$ has been written for any specific distance $r$ as:

$$
F(r, m)_{\mathrm{dB}}=\frac{\left(C_{0}-\sigma_{\mathrm{c}}\right)}{4} .
$$

The sea surface clutter radar cross-sectional area at any range $r$ is theoretically calculated as $\sigma_{\mathrm{c}}=\sigma^{0} A_{\mathrm{c}}$ where $\sigma^{0}$ is the sea surface reflectivity, and $A_{\mathrm{c}}$ is the radar cell. For a given radar distance it is possible to calculate the clutter radar cross-sectional area by using clutter models. Wind speed and direction are two variables used in sea clutter cross-section area calculations. The radar direction and altitude also participate in the calculation as intermediate values. Other parameters used in calculations are radarspecific polarization, frequency and other broadcast characteristics. As a result, if the necessary meteorological measurement data is provided, the PF can be calculated theoretically based on the distance. These values can be combined along the radar range to form a PF curve, $F(r)$. Sea clutter modelling is one of the most challenging issues of RFC problem. The dependence of the sea surface clutter model on the grazing angle for far-field radar distance, where the grazing angle is too small, has been neglected in earlier RFC estimation methods [3], [4]. On the other side, imperfectness of clutter model may affect the success of the proposed RFC estimation model negatively. Since sea clutter modelling of RFC problem has already been discussed by the researchers, it is not considered in this study, but only focused on the solution of inversion problem.

Objective Function: The atmospheric refractivity estimation models are based on the atmospheric PF and search the corresponding refractivity parameters. The atmospheric refractivity parameters, estimated by the proposed model in the global search space, are processed by the SSPE, as a candidate solution $\left(m_{\text {can }}\right)$ and a corresponding candidate PF curve $\left(F^{\mathrm{can}}\right)$ is obtained each time. An objective function computes a cost value for $F^{\text {can }}$ and the reference propagation factor, $F^{\text {ref }}$, which represents a radar observation. The cost function $f$ can be calculated using the least squares as

$$
f=\sum_{i=1}^{S}\left|F^{\mathrm{can}}\left(r_{i}, m\right)-F^{\mathrm{ref}}\left(r_{i}, m\right)\right|^{2}
$$

where $r$ is the range and $S$ is the number of samples that represent the PF curve, chosen as 144 in this study, 


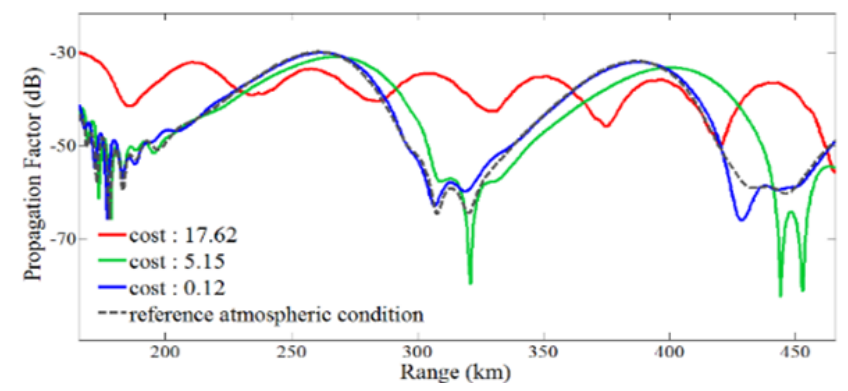

Fig. 2. Comparisons of $P F$ curves having different cost values.

The PF, obtained from radar clutter data, provides critical information for the atmospheric refractivity prediction. The main purpose of the inversion process is to find a refractivity profile that best matches to $\mathrm{PF}$ provided by radar data. It is possible to get many similar propagation results, from different refractivity profiles. Some examples of the propagation factor curves varying with the distance are shown in Fig. 2. Figure 2 also gives the reference atmospheric condition or SBD condition, chosen for this study. The cost values of the given PFs is calculated by (7), using the reference SBD case.

Testing the Performance: In this study, five different inversion algorithms are developed to estimate the $\mathrm{M}$ profile of SBD. These algorithms are ANN, sGA, aGA, sHM and aHM. In addition, sHM and aHM have 15 versions or sub-models aiming to determine the optimum contribution of ANN and GA within the hybrid structure. While measuring the overall success of competing algorithms, a popular metric, success rate is used [29]. To compare the performance of all developed models and sub-models, each one is independently executed 25 times. Then the results are compared on the basis of percentages. The acceptability of refractivity estimation is related to its use, whether it will be used for scientific or military purposes. Two control parameters, accuracy and response time, are usually recommended to terminate the estimation algorithms. Accuracy is the degree of the quality of the obtained result, which is a matter of cost. Success threshold or cost is fixed to one in this work, which means that if the cost of estimation is lower than one, then this estimation is accepted as successful and estimation process terminates. Figure 2 gives an idea about PF curves having different cost values. On the other hand, the runtime of algorithms is limited to two hours, which means that if the cost of estimation is not lower than one, but time is over, then this estimation is accepted as unsuccessful and estimation process terminates. Time limit is an issue of stability of the atmospheric conditions. If the estimation process is summarised; after estimation of $\mathrm{M}$ profiles, the corresponding PFs is produced by SSPE, after that, cost value of estimation is computed by using (7).

Simulation Data: Environmental and radar parameters used in the proposed atmospheric refractivity estimation models are given in Tab. 1. The SSPE calculation window is $470 \mathrm{~km}$ in the horizontal axis and $2000 \mathrm{~m}$ in the vertical axis. The SSPE calculation steps were chosen as $\Delta x=463 \mathrm{~m}$ in horizontal and $\Delta z=1 \mathrm{~m}$ in vertical. The

\begin{tabular}{|c|c|c|c|}
\hline Radar Freq. & Surface Type & Range Step & Altitude Step \\
\hline $1300 \mathrm{MHz}$ & Sea & $463 \mathrm{~m}$ & $1 \mathrm{~m}$ \\
\hline 3 dB Width & Elevation Angle & Antenna Height & Polarization \\
\hline $2.1^{\circ}$ & $-0.9^{\circ}$ & $1800 \mathrm{~m}$ & Horizontal \\
\hline
\end{tabular}

Tab. 1. Simulation parameters.

reason for the narrow selection of the altitude gap is that the SBD will be analyzed. The lower surface of the propagation is the sea level. The conductivity of seawater is taken as 5.208 Siemens/meter. Radar range is assumed as $470 \mathrm{~km}$ and radar altitude is chosen as $1800 \mathrm{~m}$. Signal propagation window is two-dimensional; having size of $470 \mathrm{~km}$ range $\times 2 \mathrm{~km}$ altitude. SSPE computation of this window for one environmental case (M-profile) takes approximately 17 seconds by using an Intel5 $3.5 \mathrm{GHz}, 8 \mathrm{~GB}$ DDR3 RAM computer. In the proposed refractivity profile, $M_{0}$ is taken as a constant value of 330 M-units [5]. This parameter changes very little in different atmospheric conditions and does not significantly affect propagation pattern. The atmospheric refractivity estimation performance of ANN was tested with the reference SBD case, given in Fig. 2 whose parameters are $M_{0}, M_{1}, M_{2}, H_{1}$, and $H_{2}$ and their corresponding values are chosen as 330, 360, 320, 400 and 100. This SBD environment is admittedly a simple idealized case; it represents a radar surface clutter measurement that inversion algorithms are searching for. Refractivity parameter intervals for global searches are chosen as a power of two in the simulations to facilitate binary coding in the genetic algorithm. $M_{1}$ interval is 331-394, and $M_{2}$ is $329-266$, so the total M-deficit is taken as 128 . The thickness of trapping layer, $H_{2}$ interval value is 128 . Base height search space is taken with 1024 different probability, ranging from 42 to 1065 . As a result, the size of the global search pool is obtained by computation of $64 \times 64 \times 128 \times 1024 \approx 5.36 \times 10^{8}$. The total computation time of propagation factors would take approximately 300 years by using the same computation power.

\section{Inversion with ANN and GAs}

In this part, three different atmospheric refractivity prediction models, which are based on ANN and two different GA models, are introduced. One of GA model is standard and the other is problem adapted. Problem adapted GA (aGA) is an improvement of standard GA (sGA).

ANN Model: The ANN, developed in this paper, is completely different from that of [16] in terms of both structural design and the data used in training. In addition, a nonstandard approach in data set management is performed in the new ANN model, called dynamic training data set application that improves itself continuously during the estimation process. A feed-forward neural network with one hidden layer is used as the kernel of the refractivity estimation problem. ANN structure has inputs of 144 that represent the samples of PF of radar over horizon ranges, 289 hidden layer neurons and four outputs that correspond to refractivity parameters, $M_{1}, M_{2}, H_{1}$, and $H_{2}$. 
The number of inputs is specifically determined, and it is related to a range resolution of SSPE computation. Each mile beyond the horizon in radar range is represented by one sample to represent the PF curve with enough points. A sample target-input training dataset, which represents possible atmospheric refractivity conditions, has been prepared for the training of the ANN estimation model. The selected output data set consisting of refractivity parameters for ANN training is given in Tab. 2. In this way, the output number of entities of the dataset is gathered from $7 \times 7 \times 14 \times 8=5488$ dataset. The input training dataset consisting of $F(r, m)$ is taken with the size of the $4 \times 5488$ matrix, and the corresponding output dataset is from the $144 \times 5488$ size of the matrix. Each input data is calculated by the SSPE algorithm, using the radar parameters in Tab. 1 and the refractivity parameters of reference SBD. 144 points constituting the input training set were formed from the EM propagation factor curve, from a distance of 100-244 NM over the horizon radar ranges where the surface-based effect is more clearly observed. Data rates used for training, verification, and testing are; $70 \%, 15 \%$, and $15 \%$, respectively. It was observed that the ANN training period was around 3-4 minutes, but occasionally it could be over 10 minutes depending on the samples taken from the training dataset.

Flowchart constructed for the ANN model is shown in Fig. 3. The initial data set consists of 5488 training data. However, unlike a static training data set in the conventional ANNs, in this study a novel training data set is used which is called as dynamic training data set. The dynamic training data set begins with a set of initial data having 5488 training data. This set is updated with each newly generated solution in the algorithm. Therefore, its size increases iteratively, and its database enriches with more qualified training data, coming from new estimations. Although initial training data are chosen randomly, dynamic data set contains special data, because they are estimated parameters and are mostly closer to the global solution. The

\begin{tabular}{|l|c|c|c|c|c|c|c|c|c|c|c|c|c|c|}
\hline $\boldsymbol{M}_{\mathbf{1}}$ & 334 & 342 & 350 & 358 & 366 & 374 & 382 & & & & & & & \\
\hline $\boldsymbol{M}_{\mathbf{2}}$ & 326 & 318 & 310 & 302 & 294 & 286 & 278 & & & & & & & \\
\hline $\boldsymbol{H}_{\mathbf{1}}$ & 70 & 140 & 210 & 280 & 350 & 420 & 490 & 560 & 630 & 700 & 770 & 880 & 910 & 980 \\
\hline $\boldsymbol{H}_{\mathbf{2}}$ & 15 & 30 & 45 & 60 & 75 & 90 & 105 & 120 & & & & & & \\
\hline
\end{tabular}

Tab. 2. ANN output training data set.

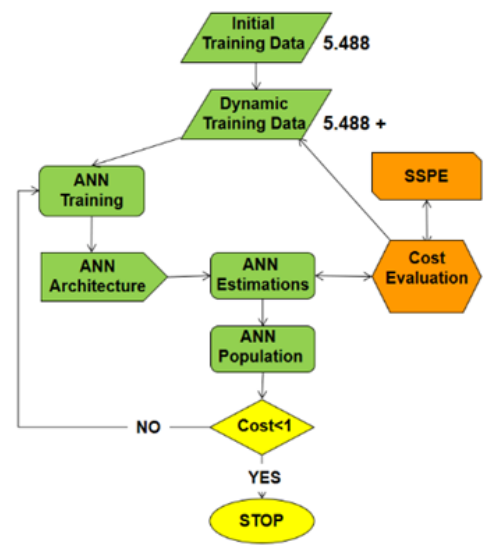

Fig. 3. Flowchart of ANN used in refractivity estimations. initial training data set composes of $5488 \mathrm{M}$ profile data and corresponding PFs which are calculated by SSPEbased EM propagation model. These calculations are performed only once in the study and the total process has taken approximately 26 hours. On the other hand, ANN training duration is about 1.5 minutes and varies due to randomly selected training, testing and verification data. The training dataset becomes dynamic after the first cycle of the algorithm and training period of ANN increases with respect to increase in number of training data. It has been observed that the training duration does never exceed 4 minutes. The success rate is obtained as $36 \%$ by running ANN estimation model 25 times. Without using dynamic training data set, the success rate for the proposed ANN model has been obtained as $10 \%$. Improvement of the success rate of the latter ANN model is a result of dynamic training data set application.

$\boldsymbol{s} \boldsymbol{G} \boldsymbol{A}$ Model: A genetic algorithm based on roulette wheel selection is used. The number of individuals in the initial population is selected as 16 for this model. Each is defined with an individual chromosome string. The algorithm presents an iterative-based evolutionary optimization procedure and produces a new generation in each iteration. Flowchart of the sGA is shown in Fig. 4. Chromosomes are represented as binary strings. The standard representation of each candidate solution is a 29-bit array; 6 bits are reserved for $M_{1}, 6$ bit for $M_{2}, 10$ bit for $H_{1}$ and 7 bit for $H_{2}$. The fitness of every individual in the population is evaluated from SSPE algorithm. Individual solutions are selected through a fitness-based process, where fitter solutions are typically more likely to be selected. In the selection phase, a fitness proportional roulette wheel is applied, so higher fitness ones may be selected more than once. Elitism is applied to two individuals in each generation. Four-point crossover is applied, with four independent variables, so each variable is exposed crossover processes independently. Crossover probability is applied as 0.75 . 12 new individuals are created in each next generation. The probability of mutation is 0.0065 , which corresponds to 3 bit for each generation. The element subjected to mutation is determined each time randomly. When sGA is applied to our refractivity problem, the success rate is obtained at $28 \%$. This is greater than the success of the ANN using the initial

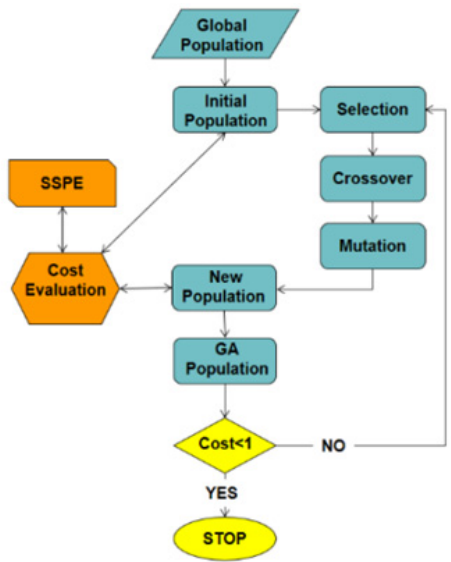

Fig. 4. Flowchart of sGA used in refractivity estimations. 
data set, but less than that of the ANN using the dynamic data set. The reason is that the algorithm gets stuck into local optimum that does not satisfy success criteria. It can be commented that GA has a good ability to control the global search space, but it is easy for GA to be mature too early, i.e., to converge too early. The algorithm has a difficulty to jump to other alternative solution areas within global search domain to find better estimations. After reaching a local solution, the only hope for jumping to a better solution is to change the chromosome of individuals using the mutation operator. The most problematic aspect of the estimation problem is to search simultaneously for four independent parameters, which constitute an individual in the population. In addition, the global research space is so large that finding a result that meets success criteria is not easy for sGA in a limited time.

$\boldsymbol{a} \boldsymbol{G} \boldsymbol{A}$ Model: A problem based genetic algorithm named as adapted Genetic Algorithm (aGA) is developed to improve the results obtained by sGA. sGA and aGA have the same flowchart given in Fig. 4. However, aGA has a mechanism to control the program flow that can detect whether there is any advancement or repetition in estimations. Problematic points that prevent the sGA model from continuing to search global solution are determined after exhausting trials. These are mostly convergence problems. Some alternative solutions can be proposed to overcome the determined problems. These alternative solutions are to change custom crossover and mutation processes in GA to decrease the stochastic side of sGA. Therefore, a stable selection mechanism is applied in aGA instead of roulette wheel selection. The best eight individuals are selected for crossover, and the second eight individuals are never given a chance to pass to next generation. Fourpoint crossover is applied again, with a probability of 0.875 . Nevertheless, elements that will pass to next generation without crossover are chosen among only the first eight elements. An operator named two-family approach is applied for crossover, and eight new individuals are created for the next generation each time. This approach corresponds to the production of two different genetic alternatives within the population to search in two different locations in global search space at the same time. Parents to be crossed are divided into two groups, and each group is subjected to crossing in itself. Thus, two different genetic traditions are emerging. If one search fails to advance, the other search can create an alternative opportunity and push the algorithm for success again. A resilient mutation depending on the fertility is applied. Mutation method and mutation probability are similar to sGA's in normal cases. However, mutation rate increases if the control mechanism detects a pause in the evolutionary process in the system. When aGA is applied to the problem, the success rate is obtained as $60 \%$. It is a noticeable improvement that the performance of aGA is nearly two times better than the performance of sGA. The performance of aGA is also better than the ANN proposed in the study. The obtained success rate can be improved further by using hybrid models, as presented in the following section.

\section{Inversion with Hybrid Models}

Two novel approaches to a hybrid model are proposed in this section; one is standard, and the other is problem adapted. Standard hybrid model (sHM) is constructed on sGA and ANN. On the other hand, the adapted hybrid model (aHM) is constructed on aGA and ANN. The algorithm of both hybrid models is constructed on the same flowchart as shown in Fig. 5. Similar to sGA, the algorithm starts with an initial population of having sixteen individuals randomly selected from the global search field in the hybrid structure. Cost evaluations of members are made via SSPE, and obtained results are added to the initial training data set of ANN. Therefore, the number of total training data reaches from 5488 to 5504 after this initial process and new data set becomes dynamic training data set. Then usual GA flow is executed by selection, crossover and mutation process, respectively. New estimations of the GA side of the flowchart generated by the first iteration are added to the training data set again. New estimation results coming from ANN side of the hybrid model are added to the dynamic training data set similarly. This addition is repeated in each iteration and dynamic training data set enriches with mostly more qualified training data coming from new estimations.

Two different populations of GA and ANN are created in the hybrid algorithm. Both populations are combined

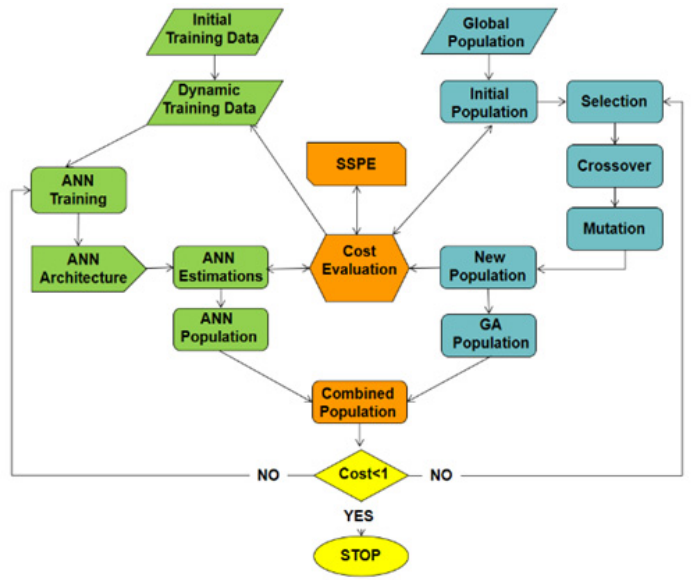

Fig. 5. Flowchart of hybrid model.
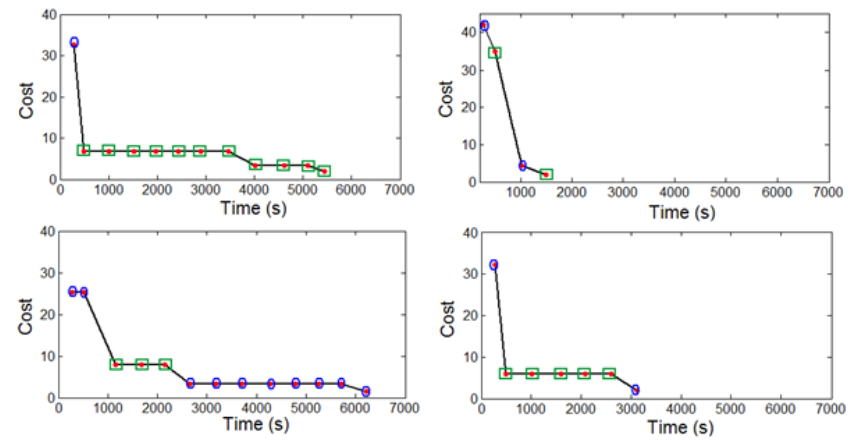

$\square$ ANN Estimation $\bigcirc$ GA Estimation

Fig. 6. Evolution of cost with contribution of GA and ANN in the HM. 
and then a new population of sixteen individuals is chosen considering their costs. If the cost of the best individual in the population is less than one, the hybrid algorithm discontinues search process. Otherwise, the second iteration starts and the process continues. The process evolution in the HM for several performed experiments can be seen in Fig. 6, following the best cost register in the algorithm. In the figure, every point shows the best estimation of overall $\mathrm{HM}$ in any iteration. The points marked with green square show ANN estimations and the points marked with blue circular show GA side estimations.

As easily seen from Fig. 6, the side of HM which makes the best estimation can alternate between ANN and GA. The differences in the figure are the reflection of the probabilistic aspect of the prediction process, which is more evident on the GA side of the model. Two algorithms cooperate perfectly to produce a better cost, like there is a labor division within the hybrid model. The dynamic training data set helps ANN to make better estimations by accepting new data continuously within overall HM. On the other hand, the cooperation of ANN and GA helps to overcome the problem of getting stuck into a local optimum that does not satisfy success criteria.

sHM Model: sHM is based on the cooperation of sGA and ANN algorithms. In this model, a combined population is created by the contributions of individuals from the ANN and GA populations. The number of combined population members is chosen to be sixteen, which is equal to the number of members in the sGA model. However, the number of individuals participating in the combined population of ANN and GA can be determined as desired. For example, if the number of members coming from the ANN is chosen as one, the number of members coming from GA must be fifteen, because the total number of members has to be sixteen. Ability to choose the amount of contribution of ANN gives the opportunity of finding the best performance point within HM. In this case, different combinations of GA and ANN can be created for the combined population. When the contribution of ANN estimations in the sHM is taken as one, the success rate is obtained as $68 \%$. In order to obtain the best success rate among different combinations, the contribution of the ANN to the combined population is increased from one to fifteen, while GA contribution is gradually decreased from fifteen to one. When the ANN contribution reaches fifteen, the contribution of GA within HM is minimal. Success rates of sHM

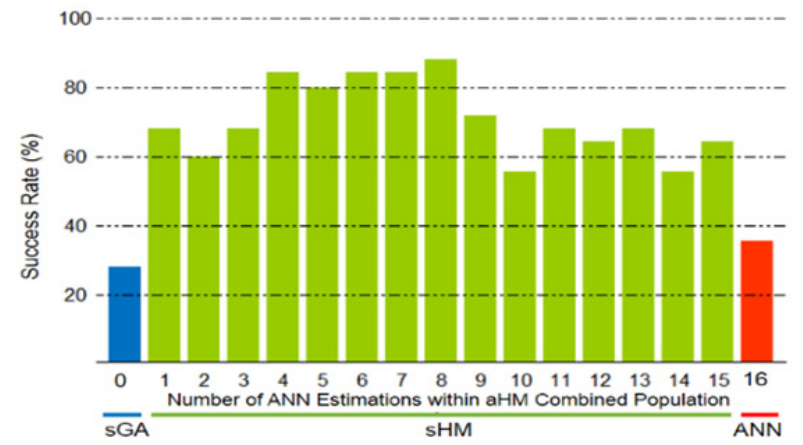

Fig. 7. Comparison of success rate of sGA, sHM's and ANN. with different ANN contributions are given in Fig. 7, including sGA and ANN success rate.

When the ANN contribution is between four and eight, the success rate of sHM exceeds $80 \%$. The success rate falls under $80 \%$ when $\mathrm{ANN}$ contributions are higher than eight, because of high cost of ANN training time. The obtained maximum success rate is $88 \%$ when ANN contribution is eight. This rate is much bigger than success rates of sGA and ANN, respectively. The fluctuations in the success rate, obtained for different ANN contributions, result from the stochastic nature of the used algorithms.

aHM Model: aHM is a cooperation of aGA and ANN algorithms. To determine the best performance point in the aHM, the optimal contributions of GA and ANN are searched in a similar way used for sHM. Success rates for a different number of ANN estimations in the aHM are given in Fig. 8. When ANN contribution within aHM is selected as one, the success rate of aHM is obtained as $80 \%$. Success rate increases gradually as the ANN contribution increases, and reaches a peak of $96 \%$ when the ANN contribution is five. The success rate falls below $80 \%$ again for nine and higher values of ANN contribution, similar to sHM estimations.

Fluctuation in results of aHM model is less than sHM results because of less probabilistic feature of aGA. Figure 9 compares the performance of two different hybrid models, standard and adapted.

If the contribution of the ANN is selected as one, the success rate for sHM and aHM are $68 \%$ and $80 \%$, respectively. The sHM reaches maximum success rate of $88 \%$ when ANN contribution is eight. On the other hand, the aHM reaches the maximum success rate of $96 \%$ when ANN contribution is 5 . Success rate of $80 \%$ is mostly

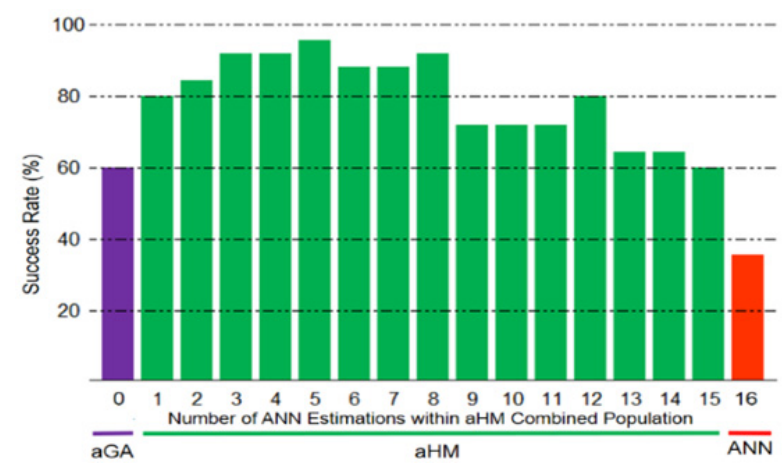

Fig. 8. Comparison of success rate of aGA, aHM's and ANN.

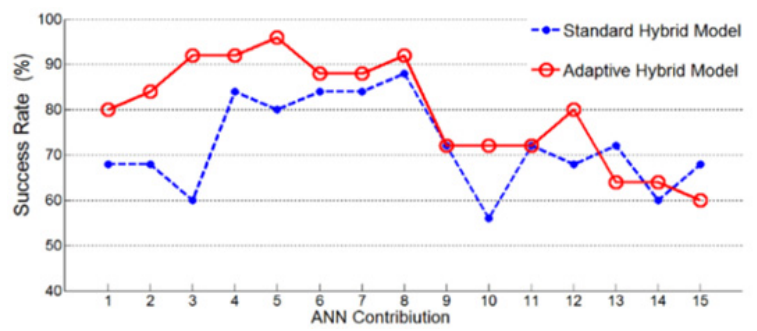

Fig. 9. Comparisons of success rate of standard and adapted approaches of the proposed hybrid model. 


\begin{tabular}{|c|c|}
\hline Method & Success Rate (\%) \\
\hline ANN & 36 \\
\hline sGA & 28 \\
\hline aGA & 60 \\
\hline sHM & 88 \\
\hline aHM & 96 \\
\hline
\end{tabular}

Tab. 3. Comparison of results.

accepted as a limit performance for evaluations of air surveillance radars. If this success criterion is also applied for measuring the success of atmospheric refractivity estimation models, the developed models in this study can be accepted as quite successful models for refractivity estimation problem. In this case, optimum ANN contribution should be taken as between $4-8$ and 1-8 for sHM and aHM, respectively. However, if the estimates will be used for especially military purposes, due to having better performance, the aHM will be more satisfactory estimation model. The highest success rate of aHM is $96 \%$ for submodel-5 (aHM-5), where the number 5 represents the amount of ANN contribution. The highest success rate of sHM is $88 \%$, for sHM-8. The maximum success rate for aGA is $60 \%$, ANN is $36 \%$ and $\mathrm{sGA}$ is $28 \%$.

\section{Conclusion}

In this paper, a novel hybrid model that is a combination of ANN and GA is proposed for the inversion of the atmospheric refractivity estimation problem by using parameters of an L-band air surveillance radar. Instead of received power signal of radar, PF parameter was used. Also, a wide-angle split step parabolic equation was used to model EM propagation. Table 3 summarizes the maximum success rates obtained from all proposed algorithms in the context of this study. The proposed hybrid models can elevate the success rate of ANN and GA in atmospheric refractivity estimations. In addition, it is observed that the dynamic training dataset application is very helpful for the improvement of ANN estimations. The results show that the success rates of aHM reach over $80 \%$ for the first eight submodels (aHM-1 to aHM-8), and maximum success is obtained as $96 \%$ for aHM-5. As a future work, instead of developing only SBD estimation, a model that can make all kinds of duct estimations can be developed. This model can be united with a clutter model and estimate atmospheric refractivity using real reflected radar signals.

\section{References}

[1] YARDIM, C. Statistical estimation and tracking of refractivity from radar clutter. Ph.D. Dissertation. University of California, San Diego (CA, USA), Electrical Engineering, 2007.

[2] YARDIM, C., GERSTOFT, P., HODGKISS, W. S. Tracking refractivity from clutter using Kalman and particle filters. IEEE Transactions on Antennas and Propagation, 2008, vol. 56, no. 4, p. 1058-1070. DOI: 10.1109/TAP.2008.919205

[3] Gerstoft, P., ROGERS, L. T., HODGKISS, W. S., et al. Refractivity estimation using multiple elevation angles. IEEE
Journal of Oceanic Engineering, 2003, vol. 28, no. 3, p. 513-525. DOI: $10.1109 /$ JOE.2003.816680

[4] GERSTOFT, P., ROGERS, L. T., KROLIK, J. L., et al. Inversion for refractivity parameters from radar sea clutter. Radio Science, 2003, vol. 38, no. 3, p. 1-22. DOI: 10.1029/2002RS002640

[5] GERSTOFT, P. An Inversion Software Package. Undersea Research Centre, La Spezia (Italy), Tech. Rep., SM-333, 1997.

[6] Gerstoft, P., GINGRAS, D. F., ROGERS, L. T., et al. Estimation of radio refractivity structure using matched-field array processing. IEEE Transactions on Antennas and Propagation, 2000, vol. 48, no. 3, p. 345-356. DOI: $10.1109 / 8.841895$

[7] YARDIM, C. GERSTOFT, P., HODGKISS, W. S. Statistical maritime radar duct estimation using hybrid genetic algorithmMarkov chain Monte Carlo method. Radio Science, 2007, vol. 42, no. 3, p. 1-15. DOI: 10.1029/2006RS003561

[8] ZHAO, X. Evaporation duct height estimation and source localization from field measurements at an array of radio receivers. IEEE Transactions on Antennas and Propagation, 2012 vol. 60, no. 2, p. 1020-1025. DOI: 10.1109/TAP.2011.2173115

[9] GRIMES, N. G., HACKETT, E. E. Examining constants in the Paulus-Jeske evaporation duct model. In United States National Committee of URSI National Radio Science Meeting (USNC-URSI NRSM). Boulder (CO, USA), 2014, p. 1-1. DOI: 10.1109/USNCURSI-NRSM.2014.6928020

[10] HOSSEINZADEH, S., SAMSUNCHI, N. The troposphere refractivity slop determination from propagation loss by the artificial neural networks. In International Symposium on Telecommunications. Tehran (Iran), 2008, p. 88-91. DOI: 10.1109/ISTEL.2008.4651277

[11] MUDROCH, M., PECHAC, P, GRABNER, M., et al. Classification and prediction of lower troposphere layers influence on RF propagation using artificial neural networks. In Proceedings of International Conference on Neural Information Processing (ICONIP08). Auckland (New Zealand), 2008, p. 893-900. DOI: 10.1007/978-3-642-02490-0_109

[12] DA SILVEIRA, R. B., HOLT, A. R. An automatic identification of clutter and anomalous propagation in polarization-diversity weather radar data using neural networks. IEEE Transactions on Geoscience and Remote Sensing, 2001, vol. 39, no. 8, p. $1177-1188$. DOI: $10.1109 / 36.942556$

[13] GRECU, M., KRAJEWSKI, W. F. Detection of anomalous propagation echoes in weather radar data using neural networks. IEEE Transactions on Geoscience and Remote Sensing, 1999, vol. 37, no. 1, p. 287-296. DOI: 10.1109/36.739163

[14] PELliCCIA, F., PACIFICI, F., BONAFONI, F., et al. Neural networks for arctic atmosphere sounding from radio occultation data. IEEE Transactions on Geoscience and Remote Sensing, 2011, vol. 49, no. 12, p. 4846-4855. DOI: 10.1109/tgrs.2011.2153859

[15] TEPECIK, C., NAVRUZ, I. A novel hybrid model for inversion problem of atmospheric refractivity estimation. $A E U$ International Journal of Electronics and Communication, 2018, vol. 84, p. 258-264. DOI: 10.1016/ j.aeue.2017.12.009

[16] TEPECIK, C., NAVRUZ, I. Solving inversion problem for refractivity estimation using artificial neural networks. In International Conference on Electrical and Electronics Engineering. Bursa (Turkey), 2015, p. 298-302. DOI: 10.1109/ELECO.2015.7394523

[17] SKOLNIK, M. Radar Handbook. 3rd ed. New York (USA): McGraw Hill. 2008. Chap. 26. ISBN: 9780071589420

[18] ANGUEIRA, P., ROMO, J. A. Microwave Line of Sight Link Engineering. Hoboken (New Jersey, USA): Wiley, 2012. P. 46. ISBN: 978-1118072738 
[19] CAIRNS-MCFEETERS, E. L. Effects of surface-based ducts on electromagnetic systems. M.S. Thesis. Naval Postgraduate School, Monterey (CA, USA), 1992, p. 1-147.

[20] DOUVENOT, R., FABBRO, V., ELIS, K. Parameter-based rules for the definition of detectable ducts for an RFC system. IEEE Transactions on Antennas and Propagation, 2014, vol. 62, no. 11, p. 5696-5705. DOI: 10.1109/tap.2014.2354680

[21] FABBRO, V., FORSTER, J., BIEGEL, G., et al. MARLENE: Mediterranean RFC and sea clutter environmental experiment. In Proceedings of the 9th European Conference of Antennas and Propagation (EuCAP). Lisbon (Portugal), 2015, p. 1-3.

[22] KARIMIAN, A., YARDIM, C., GERSTOFT, P., et al. Multiple grazing angle sea clutter modelling. IEEE Transactions on Antennas and Propagation, 2012, vol. 60, no. 9, p. 4408-4417. DOI: 10.1109/tap.2012.2207033

[23] OZGUN, O., APAYDIN, G., KUZUOGLU, M., et al. PETOOL: MATLAB-based one-way and two-way split-step parabolic equation tool for radiowave propagation over variable terrain Computer Physics Communications, 2011, vol. 182, no. 12, p. 2638-2654. DOI: $10.1016 /$ j.cpc.2011.07.017

[24] DOUVENOT, R., FABBRO, V., GERSTOFT, P., et al. A duct mapping method using least squares support vector machines. Radio Science, 2008, vol. 43, no. 6, p. 1-12. DOI: $10.1029 / 2008 \mathrm{RS} 003842$

[25] WANG, B., WU, Z. S., ZHAO, Z., et al. Retrieving evaporation duct heights from radar sea clutter using particle swarm optimization. Progress In Electromagnetics Research M., 2009, vol. 9, p. 79-91. DOI: 10.2528/PIERM09090403

[26] DOUVENOT, R., FABBRO, V., GERSTOFT, P., et al. Real time refractivity from clutter using a Best Fit approach improved with physical information. Radio Science, 2010, vol. 45, no. 1, p. 1-13. DOI: $10.1029 / 2009$ RS004137

[27] ZHANG, J. P., WU, Z. S., WANG, B. An adaptive objective function for evaporation duct estimations from radar sea echo. Chinese Physics Letters, 2011, vol. 28, no. 3, p. 1-4. DOI: $10.1088 / 0256-307 \mathrm{X} / 28 / 3 / 034301$

[28] IBEH, G. F., AGBO, G. A. Estimation of tropospheric refractivity with the artificial neural network at Minna, Nigeria. Global Journal of Science Frontier Research Interdisciplinary, 2012, vol. 12 , no. 1 , p. 8-14. ISSN: 2249-4626

[29] SIMON, D. Evolutionary Optimization Algorithms. New Jersey, (USA): Wiley, 2013, Appendix B. ISBN: 978-0-470-93741-9

\section{About the Authors...}

Cemil TEPECIK received the bachelor's degree in Electrical and Electronics Engineering from the University of Hacettepe, Ankara, the master's degree from the University of Inonu, Malatya, and the Ph.D. degree from the University of Ankara, Ankara, Turkey, in 2002, 2008, and 2018 respectively. Presently, he is working for Turkish Air Force. His research interests include air surveillance systems, antennas and wave propagation, atmospheric effects on radio waves.

Isa NAVRUZ received his B.S. degrees in Electrical and Electronics Engineering from Dokuz Eylul University, Turkey, in 1995. He received his M.S. and Ph.D. degrees in Engineering Electrical and Electronics Engineering from Gazi University. He trained as a postdoctoral research fellow at the Bio and Nano Photonics Lab - University of California, Los Angeles (UCLA) from 2011 to 2012, where he investigated the portable rapid diagnostic test reader and a cell-phone microscope for disease detection. He is currently a Prof. Dr. at the Electrical and Electronics Engineering Department of Ankara University. His current research interests include fiberoptics communication, fiber Bragg gratings and fiberoptic sensors.

O. TOLGA ALTINOZ received his B.S. from the Department of Electrical and Electronics Engineering of Baskent University in 2003. His M.S degree is from the Department of Electrical-Electronics Engineering of Hacettepe University in 2010. His Ph.D. degree is from Ankara University in 2015 from the same department. He is currently with the same department at Ankara University. He is the author or coauthor of more than 50 journal articles conference papers, books and book chapters. His current research interests include evolutionary computation, optimization, control systems, power electronics, and machine learning. 